\title{
PERCEPCIONES DE LOS JÓVENES ACERCA DE LAS ACTUACIONES Y DISCURSOS PÚBLICOS SOBRE LA VIOLENCIA DE GÉNERO EN ESPAÑA $(2013)^{1}$
}

\author{
IRENE LIBERIA VAYÁ \\ Universidad de Sevilla (España) \\ BELÉN ZURBANO BERENGUER \\ Universidad de Sevilla (España) \\ DANIEL BARREDO IBÁÑEZ \\ Universidad Laica Eloy Alfaro de Manabí (Ecuador)
}

Recibido: 13-01-2015

Aceptado: 14-05-2015

\section{Resumen}

Los jóvenes, insertos en un imaginario social sensible pero claramente desinformado, resultan preocupantes al mostrar un grado de tolerancia ante la violencia de género que es incluso mayor que en sectores poblacionales de edad más elevada, ya que al no ser capaces de identificar determinados comportamientos como sexistas -0 , llegado el caso, violentos-, no presentan discursos homogeneizados de rechazo. En ese sentido, este trabajo ha abordado la valoración de algunos estudiantes sevillanos ante los discursos políticos, judiciales y mediáticos sobre la violencia de género. Interpelados a través de la técnica de la encuesta (con campos cerrados y abiertos para su posterior tratamiento tanto cuantitativa como cualitativamente), los jóvenes interrogados han revelado una falta de confianza en las instituciones y en la justicia, así como una gran influencia de los medios de comunicación como fuente de información primera en cuanto a conocimiento sobre violencia de género. Como conclusiones principales,

1. El presente trabajo fue financiado a través de los fondos propios de los autores, así como del V Plan Propio de Investigación de la Universidad de Sevilla. 
además, se han percibido unas orientaciones generales que cuestionan como insuficientes las medidas de seguridad a las víctimas.

Palabras clave: Violencia de género; juventud; violencia contra la mujer; imaginarios colectivos; concepto social.

\begin{abstract}
Young Spanish people, embedded in a sensitive but clearly uninformed social imaginary, display a degree of tolerance for violence even higher than population of higher age. Teenagers are not able to identify certain behaviours as sexist-or, if necessary, violent-and that motivates the absence of a homogenate speech of rejection. In that sense, this work has addressed the valuation of some students of Seville to political, legal and media discourses on gender violence. Challenged through the survey technique (with closed and open fields for further quantitative and qualitative treatment), young people questioned have revealed a lack of trust in institutions and justice, as well as a large influence of the media communication as a source of first information in knowledge about gender violence. The main conclusions also have revealed some general guidelines that challenge as inadequate security measures for victims.
\end{abstract}

Key-words: Gender-based violence; youth; violence against women; collective imaginary; social concept. 


\section{Introducción}

Los últimos datos ofrecidos por el Ministerio de Sanidad, Política Social e Igualdad sobre las percepciones sociales sobre la violencia de género plantean un panorama si no desensibilizado (pues el 92\% de los españoles afirma no tolerar la violencia de género, al considerarla inaceptable), sí gravemente distorsionado, conservador y con carencias cognoscitivas relevantes. Algunos de los datos que justifican esta preocupación son los siguientes: el 36\% de los encuestados se refiere exclusivamente a la violencia física o sexual al ser preguntado por la identificación de la violencia de género; el 38\% de los españoles exculpa a los agresores porque considera que padecen una enfermedad mental; y el 35\% cree que si las mujeres sufren maltrato es porque lo consienten. Además, un porcentaje nada desdeñable considera tolerable la violencia de control (de horarios, de forma de vestir, de amistades, entre otros), (el 32\% de los hombres y el $29 \%$ de la mujeres), la desvalorización ( $10 \%$ y $8 \%$, respectivamente) y las amenazas verbales $(7 \% \text { y } 6 \%)^{2}$.

Estos y otros datos recientemente expuestos en el informe gubernamental responden perfectamente a lo que las investigadoras han descrito como «nuevo mapa de mitos de la violencia de género $»^{3}$, y que presenta evidencias como la exoneración o exculpación de los agresores bajo el convencimiento de que sufren trastornos o enfermedades psíquicas, la creencia generalizada de la influencia de los trastornos por toxicomanía en los procesos de violencia, o la consideración social de que este tipo de violencia ocurre en determinados entornos socio-económicos.

La juventud, inserta en este imaginario social sensible pero claramente desinformado, no presenta patrones de conceptualización e identificación muy alejados y es más, preocupa que su grado de tolerancia ante la violencia

2. Ministerio de Sanidad, Servicios Sociales e Igualdad. Análisis de la encuesta sobre percepción social de la violencia de género. Madrid, Ministerio de Sanidad, Servicios Sociales e Igualdad, Centro de Publicaciones, 2013. <http://goo.gl/ndDxbt>, consultado el 04-06-2014.

3. BOSCH FIOL, Esperanza y FERRER PÉREZ, Victoria A. «Nuevo mapa de mitos sobre la violencia de género en el siglo XXI». Psicothema 24, 4 (2012), pp. 548-554. 
de género sea incluso mayor que en sectores poblacionales de edad más elevada, ya que al no identificar determinados comportamientos como sexistas $-\mathrm{o}$, llegado el caso, violentos-, no presentan discursos homogeneizados de rechazo.

De hecho, en uno de los últimos estudios realizados al respecto ${ }^{4}$, el 28\% de las chicas y el 30,7\% de los chicos encuestados no consideraban que menospreciar a la pareja fuese maltrato. Como tampoco encontraban violento el control (lo que se ha venido a llamar violencia de control) en un $26,2 \%$ las chicas y en un 35\% los chicos. Incluso otros comportamientos en apariencia más fácilmente reconocibles como violentos, por ejemplo, «hacer sentir miedo», no eran interpretados como constitutivos de malos tratos por el 13,8\% de las chicas ni por el $22,8 \%$ de los chicos.

Datos más recientes 5 alertan además de la ausencia de valoración de riesgo ante conductas de maltrato, pues no son concebidas como tal. Así, el 28,1\% de los adolescentes españoles no considera una conducta de riesgo responder a un mensaje en el que se les insulte. Y ponen de manifiesto que la violencia no ha dejado de existir en este grupo poblacional que pudiera parecer -al haber crecido en un contexto con igualdad formal reconocida- que no padece violencia por razón de género: el 61,7\% asegura haber recibido mensajes con insultos. Además, el 36\% ha recibido algún mensaje que les ha hecho «sentir miedo».

Así, a las cifras de muertes se suman a estas y otras preocupantes interpretaciones sobre la violencia de género por parte de las personas más jóvenes, lo que inevitablemente lleva a dudar sobre la transmisión discursiva y conceptual de este problema social. A este respecto, debe señalarse que dicho proceso de transmisión está muy influido por los medios de comunicación, ya que en casi un $90 \%$ de las ocasiones los media se constituyen en la fuente primaria de información de la ciudadanía ${ }^{6}$, multiplicando de esta forma su importancia y responsabilidad social cuando informan sobre la violencia contra las mujeres.

En un contexto con una alta sensibilidad social con respecto al problema de la violencia contra las mujeres, pero con una falta de conocimiento y de herramientas para su identificación, la erradicación se plantea tremendamente

4. Ministerio de IGUALDAd y Universidad Complutense. Igualdad y Prevención de la Violencia de Género en la Adolescencia. Madrid, Ministerio de Sanidad, Política Social e Igualdad, Centro de Publicaciones, 2010. <http://goo.gl/nU8nZJ>, consultado el 27-11-2013.

5. Ministerio de SANidad, Servicios Sociales E IGUALDAD. Op. cit.

6. Ministerio de SAnidad, Servicios Sociales E Igualdad. Encuesta de opinión sobre la violencia de género. Madrid, Ministerio de Sanidad, Servicios Sociales e Igualdad, Centro de Publicaciones, 2011.<http://goo.gl/ijg278>, consultado el 03-12-2013. 
distante. Por ello, este trabajo pretende profundizar en el grado de conocimiento que tienen los estudiantes sobre el tema general, así como preguntarse (y preguntarles) acerca de la valoración que dan a las instituciones y la justicia en la erradicación de la violencia ya que, como es sabido, el sector poblacional joven no es usuario habitual de los servicios de protección a las víctimas, aunque es cierto que cada vez más son frecuentes las atenciones a jóvenes en una horquilla de entre 15 y 30 años.

Así lo demuestran datos como que de las casi 4.500 mujeres que llamaron al teléfono de atención para las víctimas de violencia de género (016) y cuyas edades conocemos, un 29,3\% eran jóvenes de entre 15 y 30 años ${ }^{7}$. Además, una de cada cuatro víctimas padecía violencia por parte de varones de hasta 30 años, y las y los familiares y personas allegadas a víctimas de violencia contra las mujeres que llamaron a este mismo teléfono tenían en un 47\% 30 años o menos. Las cifras según la utilización de este teléfono de ayuda -si atendemos a datos más recientes proporcionados por la Delegación del Gobierno para la Violencia de Género- se mantienen en el tiempo: entre septiembre de 2007 y diciembre de 2011 , un $28,99 \%$ de las llamadas recibidas (y cuyas edades fueron reveladas) las realizaron mujeres de 30 años o menos.

\section{Objetivos}

A partir del estado de la cuestión expuesto y las preocupaciones mostradas, esta investigación se plantea abordar los siguientes objetivos:

a. Conocer el estado de opinión sobre la violencia de género entre los estudiantes sevillanos.

b. Detectar las consideraciones de los estudiantes sevillanos sobre los poderes públicos y la protección de la justicia en cuanto a violencia de género.

c. Sondear el rol de los medios de comunicación en el conocimiento general de los estudiantes sevillanos sobre la violencia de género.

\section{Marco teórico}

En esta investigación, que se centra en la percepción de las y los jóvenes sobre los discursos y actuaciones de los poderes públicos en relación a la violencia de género, resulta imprescindible conocer qué es lo que los textos legislativos

7. Son cifras basadas en la explotación de datos de las llamadas al 016 entre el 3 de septiembre de 2007 y el 31 de diciembre de 2008, según la Delegación del Gobierno para la Violencia de Género. 
consideran como tal. En este proceso, se pondrá de manifiesto que el concepto de violencia de género que se maneja en nuestra sociedad es un concepto reduccionista. Si a esto se suma el hecho de que existen reticencias sociales y mediáticas a reconocer la violencia que sufren muchas jóvenes en nuestro país como violencia machista (a pesar del importante aumento en los últimos años del número de víctimas mortales de la violencia de género menores de 30 años), el resultado es una falta de identificación y visibilización de este grave problema en este sector poblacional. Además, ello conduce inevitablemente a una falta de concienciación que favorece el repunte de dicha violencia y evidencia el fracaso de las medidas de prevención y erradicación.

El estudio desarrollado en estas páginas parte de la idea de que el concepto de violencia de género presente en el discurso político-institucional y legislativo (en los textos jurídico-legales) posee una influencia importante en la comprensión social de este grave problema. Con el objetivo de conocer distintas definiciones legislativas de la violencia de género y elaborar un marco de referencia que sirva de ayuda a la interpretación de los datos arrojados por las encuestas que conforman la parte empírica del presente trabajo, se revisarán a continuación algunas leyes nacionales e internacionales para identificar qué entienden por violencia contra las mujeres y cuáles son -según dichos textos- sus orígenes, actores y principales manifestaciones.

\subsection{La violencia de género en las leyes}

Uno de los principales marcos discursivos a tener en cuenta a la hora de abordar la violencia de género como problema estructural de nuestras sociedades es, sin lugar a dudas, el jurídico-legal. El estudio de cómo las leyes definen este tipo de violencia hará posible el acercamiento a la conceptualización subyacente y, en parte, servirá de indicador para medir el nivel de igualdad en el que se encuentran las sociedades en el seno de las cuales se han elaborado dichas leyes.

En la presente investigación se atenderá a diferentes aspectos del concepto de violencia de género (qué prácticas y manifestaciones se incluyen en este tipo de violencia, en qué ámbitos se dan, qué actores las protagonizan, etc.) a través de tres textos legislativos de distinto ámbito de aplicación: la resolución 48/104 del 20 de diciembre de 1993 de la Asamblea General de las Naciones Unidas «Declaración sobre la eliminación de la violencia contra la mujer», la Ley Orgánica 1/2004 de Medidas de Protección Integral contra la Violencia de Género (España) y la Ley 13/2007, de 26 de noviembre, de Medidas de Prevención y Protección Integral contra la Violencia de Género (Comunidad Andaluza, España). 
Las razones de dicha selección se especifican a continuación: en primer lugar, la Declaración de 1993 de las Naciones Unidas (NNUU) «sobre la eliminación de la violencia contra la mujer» se ha escogido por ser un texto pionero que, tras más de veinte años, sigue siendo un modelo de referencia internacional. En segundo lugar, resulta fundamental por razones evidentes abordar la ley que regula las medidas de prevención y protección contra este tipo de violencia a nivel del Estado español. Por último, se toma en consideración la ley de la comunidad andaluza con el objetivo de comprobar si existen diferencias o matizaciones con respecto al texto de ámbito nacional y conseguir así una visión más global del estado de la cuestión en nuestro país. Además, el estudio empírico mediante la técnica de la encuesta que se desarrolla en este artículo se ha llevado a cabo en un centro andaluz de enseñanza secundaria.

Para comenzar, en cuanto al propio concepto de violencia de género, el texto internacional y más lejano en el tiempo, el de las Naciones Unidas (A/ RES/48/104 del 20 de diciembre de 1993), define este fenómeno como «Todo acto de violencia basado en la pertenencia al sexo femenino que tenga o pueda tener como resultado un daño o sufrimiento físico, sexual o psicológico para la mujer $\left\langle\ldots>»^{8}\right.$. Esta ley también identifica la violencia contra las mujeres como «una manifestación de relaciones de poder históricamente desiguales entre el hombre y la mujer que han conducido a la dominación de la mujer y a la discriminación en su contra por parte del hombre $»^{9}$. Es decir, la Declaración de las NNUU propone un concepto complejo de la violencia machista tanto por las manifestaciones o actos reconocidos como tal (daño o sufrimiento físico, sexual o psicológico, las amenazas de tales daños, la coacción o la privación de libertad), como por la naturaleza o causa principal de esta violencia (las relaciones de poder históricamente desiguales entre hombres y mujeres). En cuanto a los ámbitos en los que puede ejercerse este tipo de violencia, el texto de las NNUU es mucho menos restrictivo que la ley española (como se verá más adelante): en la familia, dentro de la comunidad en general (en concreto, se especifican el lugar de trabajo o las instituciones educacionales) y también se hace referencia a «La violencia física, sexual y sicológica [sic] perpetrada o tolerada por el Estado, dondequiera que ocurra» ${ }^{10}$. Con lo cual, respecto a los actores que participan en la perpetuación de esta lacra social, debe destacarse que también estos conforman un espectro más amplio que el reconocido por la ley nacional de 2004, ya que no quedan reducidos a las parejas o ex parejas de las víctimas.

8. NACIONES UNIDAS. Declaración sobre la eliminación de la violencia contra la mujer [A/ RES/48/104], 1993, p. 1.

9. Ibídem.

10. NACIONES Unidas. Op. cit., p. 2. 
Por su parte, la Ley Orgánica 1/2004 de Medidas de Protección Integral contra la Violencia de Género (España), como se ha adelantado, no define la violencia machista de forma tan compleja e inclusiva, ya que hace referencia a la violencia de género como aquella que se produce dentro del ámbito afectivo exclusivamente, en claro contraste con la idea defendida por las NNUU. En el texto español, a pesar de que en la «Exposición de motivos» se afirma que, efectivamente, la violencia de género «se dirige sobre las mujeres por el hecho mismo de serlo, por ser consideradas, por sus agresores, carentes de los derechos mínimos de libertad, respeto y capacidad de decisión ${ }^{11}$, en el artículo 1 que define el objeto de la ley, todas las agresiones masculinas hacia mujeres que se dan en otros ámbitos distintos al afectivo quedan fuera de la definición de violencia de género.

En relación con lo anterior, por lo que respecta al origen o causa de la violencia patriarcal, la ley nacional deja claro que «no es un problema que afecte al ámbito privado. Al contrario, se manifiesta como el símbolo más brutal de la desigualdad existente en nuestra sociedad $»^{12}$. En este sentido, coincide con la Declaración de las NNUU al considerar que la violencia de género es producto de la desigualdad histórica entre los sexos. Y, en la misma línea, también reconoce una amplia variedad de manifestaciones: «todo acto de violencia física y psicológica, incluidas las agresiones a la libertad sexual, las amenazas, las coacciones o la privación arbitraria de libertad $»^{13}$.

Por último, la Ley 13/2007, de 26 de noviembre, de Medidas de Prevención y Protección Integral contra la Violencia de Género (Andalucía), propone en su artículo 3 el siguiente concepto de violencia de género: «se entiende por violencia de género aquella que, como manifestación de la discriminación, la situación de desigualdad y las relaciones de poder de los hombres sobre las mujeres, se ejerce sobre éstas por el hecho de serlo» ${ }^{14}$. Es decir, la ley autonómica andaluza coincide tanto con la normativa nacional como con el texto de las NNUU en la naturaleza de la violencia contra las mujeres y, en términos generales, en su definición.

Sin embargo, en cuanto a las manifestaciones de dicha violencia, la ley andaluza es más explícita e incluye un mayor número de actos de violencia

11. EsPañA. «Ley Orgánica 1/2004, de 28 de diciembre, de Medidas de Protección Integral contra la Violencia de Género». Boletín Oficial del Estado 313 (2004), p. 42166.

12. Ibídem.

13. ESPAÑA. Op. cit., p. 42168.

14. AndaluCíA. «Ley 13/2007, de 26 de noviembre, de Medidas de Prevención y Protección Integral contra la Violencia de Género». Boletín Oficial de la Junta de Andalucía, $247(2007)$, p. 20 
que la ley estatal, ya que además de la física, la psicológica o la sexual, también habla de la violencia económica, «que incluye la privación intencionada, y no justificada legalmente, de recursos para el bienestar físico o psicológico de la mujer y de sus hijas e hijos o la discriminación en la disposición de los recursos compartidos en el ámbito de la convivencia de pareja» ${ }^{15}$.

En lo referente a los actores, además de considerar como violencia machista aquella que se ejerce sobre la mujer por parte de «quien sea o haya sido su cónyuge o por quien esté o haya estado ligado a ella por análoga relación de afectividad, aun sin convivencia ${ }^{16}$, también se hace referencia explícita en la ley andaluza a los actos de violencia «ejercidos por hombres en su entorno familiar o en su entorno social y/o laboral $»^{17}$.

Para terminar y a modo de resumen, facilitamos a continuación una tabla comparativa de las tres leyes sobre violencia de género analizadas en la que se esquematizan las manifestaciones, actores y origen de este tipo de violencia según lo expuesto en dichos textos legislativos:

Fig. 1

Análisis comparativo de las leyes sobre violencia de género

\begin{tabular}{|c|c|c|c|}
\hline & NNUU (1993) & España (2004) & Andalucía (2007) \\
\hline $\begin{array}{l}\text { Tipos/ } \\
\text { manifestaciones } \\
\text { de la violencia } \\
\text { reconocidos }\end{array}$ & $\begin{array}{l}\text { Física, sexual } \\
\text { y psicológica. } \\
\text { Amenazas, } \\
\text { coacción, } \\
\text { privación de } \\
\text { libertad. }\end{array}$ & $\begin{array}{l}\text { Física, sexual y } \\
\text { psicológica. Amenazas, } \\
\text { coacción, privación de } \\
\text { libertad. }\end{array}$ & $\begin{array}{l}\text { Física, psicológica, } \\
\text { económica, sexual y } \\
\text { abusos sexuales Amenazas, } \\
\text { coerción, privaciones } \\
\text { arbitrarias de libertad. }\end{array}$ \\
\hline $\begin{array}{l}\text { Actores de la } \\
\text { violencia } \\
\text { (victimarios } \\
\text { reconocidos) }\end{array}$ & $\begin{array}{l}\text { Familia, } \\
\text { comunidad, } \\
\text { estado }\end{array}$ & $\begin{array}{l}\text { Cónyuges o ex } \\
\text { cónyuges («o de } \\
\text { quienes estén o hayan } \\
\text { estado ligados a ellas } \\
\text { por relaciones similares } \\
\text { de afectividad, aun sin } \\
\text { convivencia») }\end{array}$ & $\begin{array}{l}\text { Cónyuges o ex cónyuges } \\
\text { («quien sea o haya sido } \\
\text { su cónyuge o por quien } \\
\text { esté o haya estado ligado } \\
\text { a ella por análoga relación } \\
\text { de afectividad, aun sin } \\
\text { convivencia») y hombres } \\
\text { del entorno familiar, social } \\
\text { y/o laboral de la víctima. }\end{array}$ \\
\hline $\begin{array}{l}\text { Origen de la } \\
\text { violencia }\end{array}$ & $\begin{array}{l}\text { Pertenencia al } \\
\text { sexo femenino }\end{array}$ & $\begin{array}{l}\text { Discriminación y } \\
\text { desigualdad en las } \\
\text { relaciones de poder }\end{array}$ & $\begin{array}{l}\text { Pertenencia al sexo } \\
\text { femenino }\end{array}$ \\
\hline
\end{tabular}

Fuente: elaboración propia

15. Ibídem.

16. Ibídem.

17. Ibídem.

Feminismo/s 25, junio 2015, pp. 159-182 


\section{Metodología}

Este trabajo, de carácter exploratorio, constituye la primera etapa de un proyecto más extenso con el que los autores se fijaron analizar de forma integral las percepciones de los estudiantes andaluces sobre la violencia de género.

En esta primera de las etapas, la falta de financiación y, por tanto, la escasez de recursos ${ }^{18}$, son algunos de los factores que motivaron la elección de una metodología basada en una encuesta, una técnica cuantitativa muy utilizada en ámbitos científicos como el de la Psicología ${ }^{19}$ y en general en las Ciencias Sociales ${ }^{20}$. El cuestionario se preparó en función de la experiencia acumulada en tres tipos de fuentes:

Fig. 2

Fuentes utilizadas para diseñar las variables del proyecto

\begin{tabular}{|c|c|c|}
\hline Fuentes institucionales & Estudios de los autores & Estudios de otros autores \\
\hline $\begin{array}{l}\text { Ministerio de Igualdad y } \\
\text { Universidad Complutense } \\
\text { (2010) }\end{array}$ & $\begin{array}{l}\text { Zurbano (2012; 2014); } \\
\text { Zurbano y Liberia (2014); } \\
\text { Barredo y Oller (2012); } \\
\text { Zurbano, Liberia y Campos } \\
(2015)\end{array}$ & $\begin{array}{l}\text { Gutiérrez - Ravé (2009); } \\
\text { bitácora «Lo siento, no } \\
\text { volverá a pasar. Una } \\
\text { investigación sociológica } \\
\text { sobre la violencia de género } \\
\text { en España» }{ }^{21}\end{array}$ \\
\hline
\end{tabular}

Fuente: elaboración propia

El cuestionario incluía un total de 30 preguntas, abiertas y cerradas, con las cuales se intentó cuantificar las opiniones de un grupo homogéneo, pero también recoger las claves aportadas por esos mismos individuos ${ }^{22}$. La fase

18. En tanto que, como se aclaró desde el inicio, el proyecto contó únicamente con los fondos propios de los investigadores, así como del V Plan Propio de Investigación de la Universidad de Sevilla.

19. Coolican, Hugh. Métodos de investigación y estadística en Psicología. México, El Manual Moderno, 1994

LozAno, José Carlos. Teoría e investigación de la comunicación de masas <2. ${ }^{a}$ edición>. México, Pearson Educación, 2007.

20. Ander-EGG, Ezequiel. Técnicas de Investigación Social <23. a edición>. Buenos Aires, Magisterio del Río de La Plata, 1993.

21. Consultada a efectos académicos en: <http://goo.gl/naZONg> (fecha de última consulta: 07/05/2014).

22. Las respuestas del cuestionario, según sus bloques conceptuales, han sido repartidas en el presente trabajo, así como en Libera, Irene; ZuRBANo, Belén y BARREDO, Daniel. «El concepto social de la violencia de género en España. Aproximaciones a los imaginarios 
de pretest del cuestionario se desarrolló en el mes de abril de $2013^{23}$. En ese mismo mes se inició la recolección de datos de manera presencial, un método que ha sido criticado por ser «intrusivo y reactivo $»^{24}$, pero que sin embargo presenta algunas ventajas, como el ahorro de los costes o la fiabilidad de la participación, principales desventajas del levantamiento de información a través de llamadas telefónicas, o del envío de los cuestionarios aprovechando Internet.

La ausencia de financiación provocó que se rechazara la búsqueda de un muestreo probabilístico, y se optara por un muestreo por conveniencia o accidental ${ }^{25}$. En los estudios que emplean este tipo de muestreo se desaconseja efectuar generalizaciones ${ }^{26}$, en tanto que las opiniones recogidas no son una representación de una población general. En este caso, las opiniones que se ofrecen pertenecen, entonces, a una investigación de carácter «intensivo» ${ }^{27}$, al concentrarse en codificar las percepciones de un grupo de encuestados sevillanos de varios ciclos formativos de grado medio y grado superior de formación profesional.

Por último, los datos se analizaron y se contextualizaron en el primer semestre de 2014, únicamente de modo descriptivo, ya que en los proyectos que se trazan a partir de etapas iniciales de carácter exploratorio se pretende, sobre todo, «<...> (1) El diagnóstico de una situación, (2) Selección de Alternativas, y (3) El descubrimiento de nuevas ideas ${ }^{28}$.

\subsection{Descripción de la muestra}

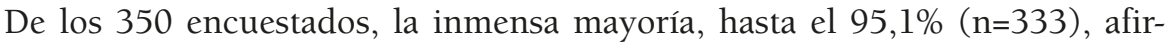
maba que no tenía hijos:

de jóvenes estudiantes (2013)», Revista interdisciplinaria Estudios de Género de El Colegio de México 2 (2015) (en impresión).

23. Intervinieron en esta fase el Dr. Javier Herrero (Universidad de Salamanca, España), el Dr. Martín Oller Alonso (Instituto de Altos Estudios Nacionales, Ecuador) y los propios investigadores participantes.

24. BERnARD, H. Russell. Social Research Methods. Qualitative and Quantitative Approaches $<2$. $^{a}$ edición $>$. Londres, Sage, 2013, p. 220.

25. CoOliCan, Hugh. Op. cit.

26. HeRnÁNDEZ, Óscar. Estadística elemental para Ciencias Sociales. San José, Universidad de Costa Rica, 2004.

27. ARIAS, Fidias G. El Proyecto de Investigación. Introducción a la metodología científica <quinta edición>. Caracas, Episteme, 2006, p. 31.

28. ABREU, José Luis: «Hipótesis, Método y Diseño de Investigación». Daena: International Journal of Good Conscience 7, 2 (2012), p. 192. 
Fig. 3

Descripción de la muestra empleada por sexo y edades (2013)

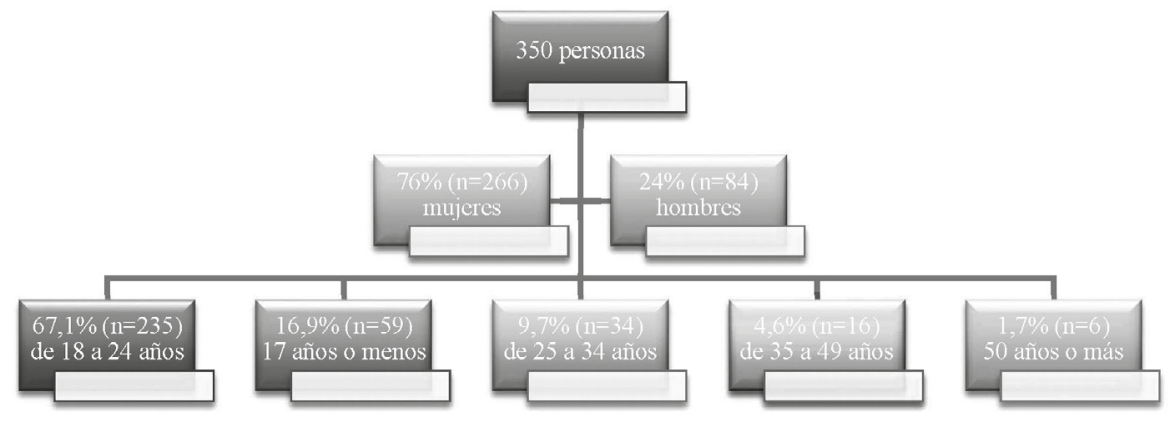

Fuente: elaboración propia

Según puede observarse en el gráfico anterior, el grupo poblacional más elevado integrante de la muestra oscilaba entre los 18 y los 24 años, seguido a mucha distancia de los encuestados con 17 años o menos. Si existen tendencias minoritarias -representadas por los estratos de 35 o más años- es por la diversidad reinante en las aulas españolas, en donde no es difícil encontrar a individuos que alcancen esas edades.

\section{Resultados}

Los resultados se han agrupado alrededor de los grandes objetivos de este trabajo: la evaluación de las opiniones generales sobre la violencia de género, las consideraciones sobre los poderes públicos y la protección de la justicia, y el rol desempeñado por los medios de comunicación.

\subsection{Opiniones generales sobre la violencia de género}

Dentro del grupo de estudiantes encuestados, hemos hallado diversas tendencias para aludir a la violencia contra las mujeres: la mayor parte de las mujeres encuestadas, con un $24,6 \%(n=86)$, prefiere definir esta problemática como «maltrato», mientras que el $8,6 \%$ de los hombres $(n=30)$ prefiere denominarlo como "violencia de género»; en cualquier caso, ambas tendencias siguen trayectorias similares, si bien casi el triple de mujeres emplea la construcción «violencia machista» frente a los hombres, según puede observarse en el gráfico siguiente: 
Fig. 4

Términos más adecuados (en \%) para referirse a la violencia contra las mujeres, según los estudiantes encuestados (2013)

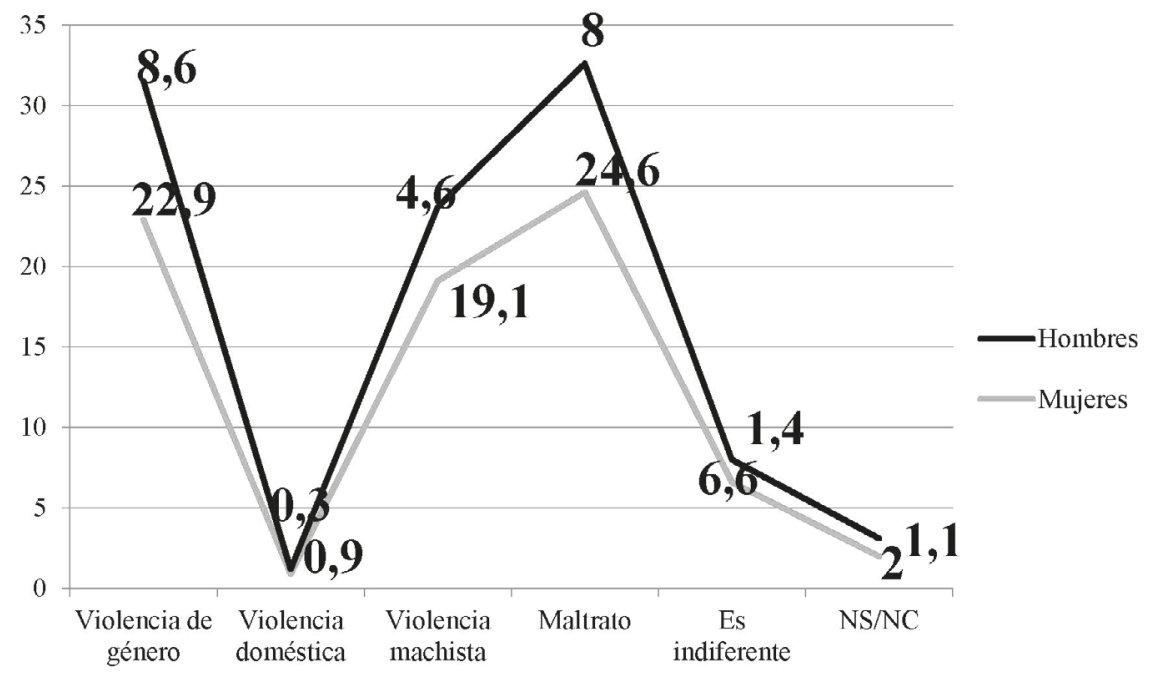

Fuente: elaboración propia

Un 86,9\% ( $\mathrm{n}=304)$ de los encuestados considera que la violencia de género es un problema de toda la sociedad, y aconseja que haya una implicación colectiva, de los que el 66\% ( $n=231)$ son mujeres y el 20,9\% ( $n=73)$ hombres. Solo un $7,7 \%(n=27)$ de los estudiantes afirma que la violencia de género es un problema exclusivamente de las parejas y de las familias donde se dan casos de malos tratos, de los que el 5,7\% ( $n=20)$ son mujeres y el $2 \%(n=7)$ hombres. Y en esta pregunta, un 5,4\% ( $n=19)$ de los encuestados no se ha decantado por ninguna de estas opciones, sino que ha preferido abstenerse.

Al cuestionar a los estudiantes la pregunta ihas ejercido alguna vez violencia contra tu pareja?, solo el 3,1\% $(n=11)$ de los encuestados declara haber ejercido alguna vez violencia contra sus parejas, de los que el 2,9\% ( $n=10)$ son mujeres y el 0,3\% ( $n=1)$ hombres. El 94\% ( $n=329)$ de los encuestados niega haber empleado violencia contra sus parejas, siendo el 70,9\% ( $n=248)$ mujeres y el 23,1\% ( $n=81)$ hombres. Y un 2,9\% $(n=10)$ de los estudiantes no ha querido aportar su experiencia, de los que el $2,3 \%(n=8)$ son mujeres y el $0,6 \%(n=2)$ hombres.

También hemos cuestionado a los estudiantes sevillanos sobre cómo actuarían en caso de ser testigos de algún acto de violencia de género; el 
$71,7 \%(\mathrm{n}=251)$ sostiene que la violencia contra las mujeres es un delito que debe denunciarse siempre, de los cuales el $56,3 \%(n=197)$ son mujeres y el $15,4 \%(n=54)$ hombres. Un 20,3\% $(n=71)$ de los estudiantes afirma que si conoce a la pareja en cuestión primero intentaría hablar para ayudar a solucionar el conflicto, y en el caso de que no lo consiguiera, entonces acudiría a la policía; el 14\% $(n=49)$ de las mujeres se decanta por esta opción, frente al $6,3 \%(n=22)$ de los hombres. Un 3,4\% ( $n=12)$ de los encuestados afirma que lo que ocurre dentro de una pareja es privado y nadie tiene derecho a meterse en sus asuntos porque no se conocen los detalles ni las motivaciones, de los que el 2,3\% ( $n=8)$ son mujeres y el 1,1\% (n=4) hombres. Solo un 0,6\% (n=2) de los estudiantes piensa que hay que denunciar a la víctima o al agresor solo si son personas del propio entorno, siendo un $0,3 \%(n=1)$ mujeres y un $0,3 \%$ $(\mathrm{n}=1)$ hombres $)$. Por último, un $4 \%(\mathrm{n}=14)$ de los estudiantes se ha abstenido en aportar su opinión, de los que el 3,1\% (n=11) son mujeres y el 0,9\% (n=3) hombres.

En cuanto a las actuaciones de los estudiantes encuestados ante un caso de violencia de género, el 64\% opta por llamar a la policía, si bien casi dos de cada diez estudiantes ignoran qué harían en ese caso hipotético:

Fig. 5

Actuaciones de los encuestados ante un caso de violencia de género (2013)

\begin{tabular}{|l|c|c|c|c|c|c|}
\hline & \multicolumn{2}{|c|}{ Mujeres } & \multicolumn{2}{c|}{ Hombres } & \multicolumn{2}{c|}{ Total } \\
\hline & $\%$ & $\mathrm{~N}$ & $\%$ & $\mathrm{~N}$ & $\%$ & $\mathrm{~N}$ \\
\hline $\begin{array}{l}\text { Avisar a alguien de } \\
\text { mi entorno }\end{array}$ & 9,4 & 33 & 2,3 & 8 & 11,7 & 41 \\
\hline Llamar a la policía & 51,1 & 179 & 12,9 & 45 & 64 & 224 \\
\hline $\begin{array}{l}\text { Acudir a la vivienda } \\
\text { donde oigo la } \\
\text { agresión }\end{array}$ & 0,9 & 3 & 3,4 & 12 & 4,3 & 15 \\
\hline Nada & 1,1 & 4 & 0,9 & 3 & 2 & 7 \\
\hline No lo sé & 10,3 & 36 & 3,4 & 12 & 13,7 & 48 \\
\hline $\begin{array}{l}\text { No sabe / No } \\
\text { contesta }\end{array}$ & 3,1 & 11 & 1,1 & 4 & 4,3 & 15 \\
\hline
\end{tabular}

Fuente: elaboración propia

Casi 8 de cada 10 estudiantes, es decir, el 76,9\% (n=269) de los encuestados confiesan que denunciarían un caso de violencia de género al ser testigos 
presenciales, de los cuales el $57,7 \%(\mathrm{n}=202)$ son mujeres y el $19,1 \%(\mathrm{n}=67)$ hombres. Únicamente el 7,1\% $(\mathrm{n}=25)$ de los estudiantes no denunciaría un caso de violencia de género al presenciarlo, de los que el 5,1\% ( $n=18)$ son mujeres y el $2 \%(n=7)$ hombres. Un $14,3 \%(n=50)$ denunciaría el caso dependiendo de determinados factores, de los que el $11,7 \%(\mathrm{n}=41)$ son mujeres y el $2,6 \%(n=9)$ hombres. Y, finalmente, el $1,7 \%(n=6)$ de los encuestados no ha querido aportar su opinión, de los que el $1,4 \%(n=5)$ son mujeres y el $0,3 \%$ $(\mathrm{n}=1)$ hombres.

Al preguntar a los encuestados sobre una posible solución para el problema social que representa la violencia de género, hemos percibido dos grandes tendencias grupales: el 50,6\% $(n=177)$ de los estudiantes sostiene que la solución pasa por procurar unas leyes más justas, así como unas condenas más duras e íntegras; es partidario de esta opción el 40,3\% (n=141) de las mujeres y el $10,3 \%(n=36)$ de los hombres. La otra gran tendencia, que alcanza el 40\% $(n=140)$ de los encuestados, reconoce que una educación en y para la igualdad entre hombres y mujeres serviría para paliar el fenómeno de la violencia, de los que el 29,4\% ( $n=103)$ son mujeres y el 10,6\% ( $n=37)$ hombres. Solo un $3,7 \%(n=13)$ de los estudiantes se decanta por evitar el maltrato con un divorcio a tiempo, siendo un $2,6 \%(n=9)$ mujeres y un $1,1 \%(n=4)$ hombres. Un 2,9\% $(\mathrm{n}=10)$ de los encuestados aporta otro tipo de soluciones, de los que el $1,7 \%(n=6)$ son mujeres y el $1,1 \%(n=4)$ hombres. Y, finalmente, un $2,9 \%(\mathrm{n}=10)$ de los estudiantes se ha abstenido a la hora de responder a esta pregunta.

\subsection{Consideraciones sobre los poderes públicos y protección de la justicia}

Un $65,1 \%(n=228)$ de los estudiantes encuestados critica a los poderes públicos, ya que opina que esos poderes no se implican suficientemente en la problemática de la violencia de género.

El 58,3\% (n=204) de los encuestados sostiene que las mujeres solo están protegidas a veces por la justicia, de los cuales el $47,4 \%(n=166)$ son mujeres y el 10,9\% ( $n=38)$ hombres. Un 26\% ( $n=91)$ está de acuerdo con la protección brindada por la justicia a las mujeres, siendo un $14,9 \%(n=52)$ mujeres y un $11,1 \%(n=39)$ hombres. Finalmente, un $14,9 \%(n=52)$ de los estudiantes afirma que las mujeres no están correctamente protegidas, de los que el 13,4\% $(n=47)$ son mujeres y el $1,4 \%(n=5)$ hombres. En esta pregunta se ha abstenido un $0,9 \%(n=3)$ de los encuestados. 
Fig. 6

Valoración (en \%) sobre la implicación de los poderes públicos en la violencia de género, según los estudiantes encuestados (2013)

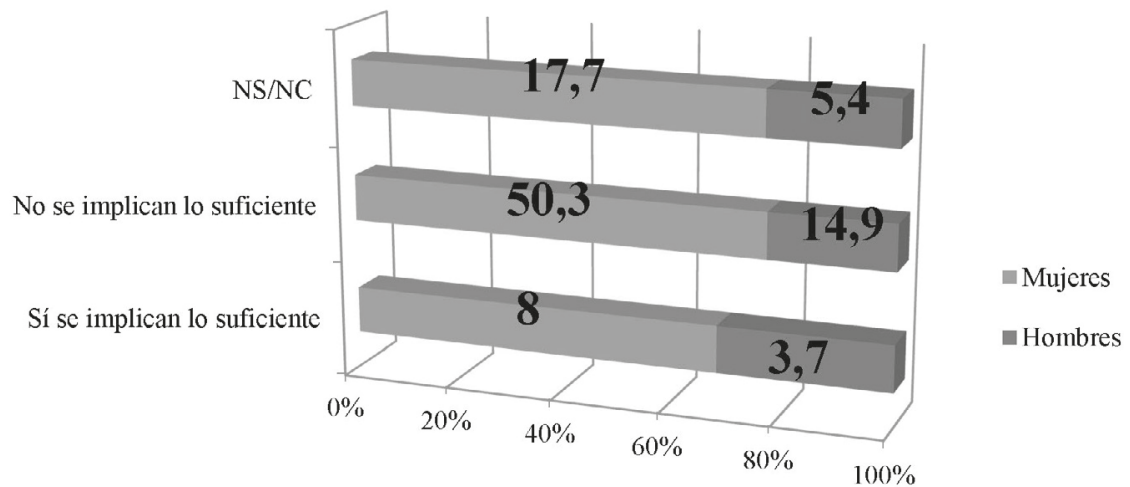

Fuente: elaboración propia

De una forma bastante similar, el 53,4\% (n=187) de los estudiantes encuestados subraya que los hombres solo están protegidos ocasionalmente por la justicia, de los cuales el 42,3\% ( $n=148)$ son mujeres y el $11,1 \%(n=39)$ hombres. Un 16,9\% $(n=59)$ del grupo está de acuerdo con la protección que reciben los hombres por parte de la justicia, de los que el 11,4\% ( $n=40)$ son mujeres y el 5,4\% ( $n=19)$ hombres. Casi tres de cada diez encuestados, es decir, el 28,9\% ( $\mathrm{n}=101)$ de los estudiantes cuestionan la protección que reciben los hombres, siendo un 22\% $(n=77)$ mujeres y un $6,9 \%$ $(n=24)$ hombres. Solo el 0,9\% (n=3) de los encuestados se abstuvo en esta pregunta.

El siguiente gráfico ilustra las posturas globales obtenidas por el grupo sobre las opiniones que tienen acerca de la protección que ofrece la justicia tanto a hombres como a mujeres: 
Fig. 7

Percepciones globales (en \%) de los estudiantes encuestados ante la protección que ofrece la justicia (2013)

¿Las mujeres y los hombres están protegidos por la justicia?

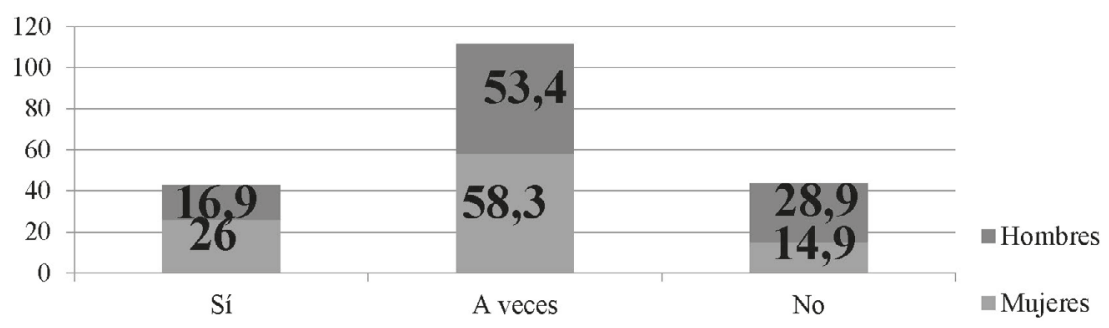

Fuente: elaboración propia

El 58,3\% (n=204) de los encuestados considera que la orden de alejamiento protege en parte a la mujer maltratada, pero no al $100 \%$, de los cuales un $42,3 \%(n=148)$ son mujeres y un $16 \%(n=56)$ hombres. Un 39,1\% ( $n=137)$ sostiene que la orden de alejamiento no sirve para nada, siendo un $31,7 \%$ $(n=111)$ mujeres y un 7,4\% ( $n=26)$ hombres. Solo un $1,7 \%(n=6)$ está absolutamente de acuerdo en que la efectividad de la orden de alejamiento protege a la mujer maltratada, de los que un $1,4 \%(n=5)$ son mujeres y un $0,3 \%(n=1)$ hombres. Únicamente un $0,3 \%(\mathrm{n}=3)$ de los encuestados ha decidido no aportar su opinión a esta pregunta.

\subsection{El rol de los medios}

Al cuestionar a los estudiantes sobre el papel que cumplen los medios de comunicación con respecto a la violencia de género, el 70,9\% ( $\mathrm{n}=248)$ escoge la opción que subraya la importancia de esos mediadores simbólicos, al reconocer que: Son muy importantes porque casi toda la información que tenemos acerca de este tipo de violencia nos llega a través de los medios. Por ello, tienen una gran responsabilidad y el deber de informar correctamente y de denunciar todos los casos de violencia de género; de ese elevado porcentaje, el 52,6\% $(\mathrm{n}=184)$ son mujeres y el 18,3\% ( $\mathrm{n}=64)$ hombres. Un $15,1 \%(\mathrm{n}=53)$ de los estudiantes afirma que los medios de comunicación no juegan ningún papel en este problema porque se limitan a informar, de los cuales el 12\% ( $n=42)$ son mujeres y el 3,1\% ( $n=11)$ hombres Un 11,1\% (n=39) de los encuestados 
denuncia la mala praxis de los medios, ya que: Tienen una influencia negativa porque dan ideas nuevas a los maltratadores $y$, aunque sea de manera involuntaria, les incitan a ejercer más violencia; de ese porcentaje, un $9,4 \%(n=33)$ son mujeres y un $1,7 \%(n=6)$ hombres. En esta pregunta, se ha abstenido un 2,9\% $(\mathrm{n}=10)$ de los encuestados.

Casi seis de cada diez estudiantes, o sea el 56,9\% (n=199), consideran que no se habla lo suficiente de la violencia de género:

Fig. 8

Percepciones (en \%) sobre el grado de actualidad de la violencia de género, según los estudiantes encuestados (2013)

La violencia de género es un tema del que se habla...

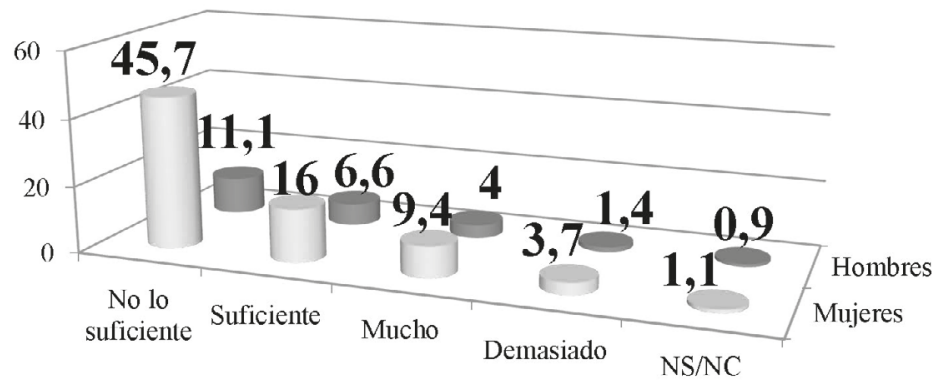

Fuente: elaboración propia

El 55,7\% ( $\mathrm{n}=195)$ de los estudiantes reconoce que sí que toma conciencia ante las situaciones de malos tratos a mujeres que aparecen en los medios de comunicación, de los que el 41,1\% ( $n=144)$ son mujeres y el 14,6\% ( $n=51)$ hombres. Un 38\% de los encuestados afirma tomar conciencia sobre este problema solo a veces, de los que el 30,3\% ( $n=106)$ son mujeres y el 7,7\% ( $n=27)$ hombres. Solo un $4,3 \%(n=15)$ exclama no tomar conciencia ante las situaciones de malos tratos a mujeres difundidas por los medios de comunicación, siendo un $3,1 \%(\mathrm{n}=11)$ mujeres y un $1,1 \%(\mathrm{n}=4)$ hombres. Y, por último, un $2 \%(\mathrm{n}=7)$ de los encuestados se ha abstenido a responder esta pregunta.

Los medios de comunicación, y en menor medida la información adquirida a través de Internet, son los principales actores que fomentan la construcción de los imaginarios sobre violencia de género en los estudiantes encuestados: 
Fig. 9

Principales actores en la construcción de los imaginarios sobre violencia de género, según los estudiantes encuestados (2013)

\begin{tabular}{|l|c|c|c|c|c|c|}
\hline & \multicolumn{2}{|c|}{ Mujeres } & \multicolumn{2}{c|}{ Hombres } & \multicolumn{2}{c|}{ Total } \\
\hline & $\%$ & $\mathrm{~N}$ & $\%$ & $\mathrm{~N}$ & $\%$ & $\mathrm{~N}$ \\
\hline $\begin{array}{l}\text { Experiencias en } \\
\text { pareja }\end{array}$ & 3,4 & 12 & 0,3 & 1 & 3,7 & 13 \\
\hline Charlas entre amigos & 0,9 & 3 & 0,9 & 3 & 1,7 & 6 \\
\hline $\begin{array}{l}\text { Charlas con los } \\
\text { padres }\end{array}$ & 2,6 & 9 & 0 & 0 & 2,6 & 9 \\
\hline $\begin{array}{l}\text { Las relaciones que } \\
\text { he visto en mi casa/ } \\
\text { familia }\end{array}$ & 2 & 7 & 0,9 & 3 & 2,9 & 10 \\
\hline Trabajos de clase & 1,7 & 6 & 0 & 0 & 1,7 & 6 \\
\hline $\begin{array}{l}\text { Explicaciones de los } \\
\text { profesores }\end{array}$ & 2,3 & 8 & 0,9 & 3 & 3,1 & 11 \\
\hline $\begin{array}{l}\text { Medios de } \\
\text { comunicación }\end{array}$ & 39,7 & 139 & 13,7 & 48 & 53,4 & 187 \\
\hline Internet & 9,4 & 33 & 2,9 & 10 & 12,3 & 43 \\
\hline $\begin{array}{l}\text { No sabe / No } \\
\text { contesta }\end{array}$ & 14 & 49 & 4,6 & 16 & 18,6 & 65 \\
\hline
\end{tabular}

Fuente: elaboración propia

Sorprende el hecho de que, según la tabla anterior, casi dos de cada diez estudiantes no saben cuál de los ámbitos ofrecidos tiende a impactar más fuertemente en su cultura sobre violencia de género.

\section{Discusión y principales conclusiones}

La violencia de género es una realidad socialmente visible en España y frente a la que la mayoría de los ciudadanos y ciudadanas, incluyendo a la juventud, se encuentra sensibilizada. De hecho, los datos aquí presentados reflejan poca incidencia de esta violencia en la muestra: un porcentaje ínfimo de los encuestados declara haber ejercido violencia. Es sin embargo destacable, por un lado, que sean más mujeres que hombres las que declaren haber ejercido violencia contra sus parejas siendo el objeto de la encuesta la violencia de género (lo que denota una distorsión conceptual); y, de otro que, vista la percepción social generalizada que se tiene de este problema, cabe preguntarse 
si el dato sobre violencia ejercida o recibida es real o, por el contrario, está influenciada (y no identificada) por los marcos conceptuales subyacentes.

A este respecto y en relación con algunos de los resultados expuestos en el apartado anterior, cabe recordar (se han visto reflejadas precisamente en el citado epígrafe) tres de las principales preguntas de investigación que fueron planteadas al comienzo de este trabajo y que han servido de guía y encauzado este proyecto: a) ¿Cuáles podrían ser los principales actores que tienden a construir el imaginario sobre la violencia de género de los estudiantes encuestados?; b) Los estudiantes y las estudiantes de Sevilla, ¿manifiestan, a grandes rasgos, diferentes percepciones sobre la protección que ofrece la justicia? y c) ¿Cuáles serían las actuaciones más habituales de las y los estudiantes sevillanos ante un caso de violencia de género?

En cuanto a la primera pregunta, ya se ha hecho referencia a la posible distorsión del concepto de violencia de género que manejan las y los estudiantes encuestados, vista su respuesta a la pregunta sobre si alguna vez han ejercido violencia sobre sus parejas. Además, en los párrafos que siguen, se hablará nuevamente del importante papel que juegan los medios de comunicación en la concepción que la población tiene de este grave problema social. Y en relación con esta cuestión, se encuentra otro de los elementos más destacables de la investigación desarrollada en estas páginas: la crítica a las instituciones que hacen las y los estudiantes sevillanos respecto a las medidas para luchar contra la violencia de género (esto tiene que ver con la segunda de las preguntas de investigación planteadas). El 65\% de la población encuestada no encuentra suficiente la labor que realizan y el $60 \%$ no percibe, además, que las mujeres víctimas estén siempre protegidas. Estos datos -junto con otros aportados en los resultados- evidencian una clara falta de confianza por parte de la juventud estudiantil en las instituciones y en la justicia española con respecto a la violencia ejercida contra las mujeres. De hecho, ni siquiera valoran como adecuadas las medidas existentes en la actualidad, como la orden de alejamiento. Cabe destacar a este respecto que al finalizar 2008, la proporción de mujeres víctimas de 15 a 30 años con protección judicial activa era del 39,2\% ${ }^{29}$.

Esto no quiere decir que los jóvenes pretendan suplir las actuaciones del Estado (y se entra aquí de lleno en la tercera pregunta de investigación planteada), pero a tenor de los datos y a pesar de considerarse mayoritariamente un asunto a denunciar en todos los casos, hay aún porcentajes importantes de estudiantes cuya acción en caso de presenciar una agresión no se

29. ZuRbano, Belén; Liberia, Irene y CAmpos, Beatriz. «Concepto y representación de la violencia de género. Reflexiones sobre el impacto en la población joven». Oñati SocioLegal Series. OSLS 5, 2 (2015) (En impresión). ISSN: 2079-5971. 
correspondería con la que demandan las fuerzas públicas. Así, el 20\% de los jóvenes interrogados sugiere que mediaría, que iniciaría charlas para solventar el conflicto, y un 16\% que no sabría qué hacer o que no haría nada; es fundamental reflejar este dato, pues supone una contradicción entre el panorama sensibilizado, intolerante y crítico para con la situación actual de violencia contra las mujeres en España, y los comportamientos que aseguran que tendrían ante la presencia de una agresión violenta. Contradicción asimilable al contexto de crecimiento y desarrollo de estos jóvenes que, por un lado, conocen de la igualdad formal en la que se hallan, pero por el otro sufren discriminaciones y violencia a diario ${ }^{30}$.

Volviendo a la cuestión de los actores sociales que más influyen en el imaginario sobre la violencia contra las mujeres que manejan las y los jóvenes encuestados, resulta obvio que los medios de comunicación son agentes decisivos en la estructuración de dicho imaginario ya que, como reconocen las y los estudiantes, suponen la fuente de información primera sobre este problema (seguida de Internet). Dato que debe hacer reflexionar sobre las representaciones que se hacen de esta violencia, los canales que se utilizan y las metodologías para su acercamiento -en su complejidad- a la sociedad y a los jóvenes en particular, ya que es claro a tenor de los datos aquí expuestos que los medios de comunicación afectan de manera notable en la toma de conciencia sobre el problema de la violencia de género.

Visto que los medios se configuran como la fuente de información primera y fundamental para la población joven estudiante (aunque no solo ${ }^{31}$, como ya se ha señalado en la introducción de este trabajo), es destacable, en contraposición, la poca formación al respecto que algunos de estos jóvenes presentan. En esta parte del cuestionario, de tipo abierto, hay quienes han restado importancia a la responsabilidad que tienen los medios en las influencias en la conducta social, ya que estos «sólo informan», denotando así una gran falta de conocimiento y herramientas en la decodificación de los mensajes mediáticos. Si bien hay que destacar lecturas muy positivas -marcadamente femeninas- que aluden al peligro de los mensajes que emiten los medios porque «dan ideas» a los agresores. Esta última idea coincide con lo

30. De Miguel, Ana. «Feminismo y juventud en sociedades formalmente igualitarias». Revista de Estudios de Juventud 83 (2008), pp. 29-45.

31. Ministerio de SANidAd, Servicios Sociales e Igualdad. Encuesta de opinión sobre la violencia de género. Op. Cit. 
que otros investigadores ya anunciaron como riesgos o efectos de las noticias sobre agresiones a mujeres ${ }^{32}$.

En resumen, los resultados aquí obtenidos evidencian que el fenómeno de la violencia de género es conocido y rechazado y que la mayor parte del conocimiento que se tiene sobre él es resultado de las influencias mediáticas. Las y los jóvenes son críticos con las medidas institucionales y judiciales implementadas y valoran como insuficientes las medidas de seguridad ofrecidas a las víctimas. Además, persiste aún la idea de la violencia de género como disputa o conflicto entre pares entre los que se puede mediar u obtener acuerdo, nada más alejado de la realidad del problema de la violencia de género, y cuya consideración conceptual va a influir (como se ha demostrado en estas páginas) sobre las actitudes a adoptar, por ejemplo, ante un caso de violencia real.

Estos datos y conclusiones deben hacernos reflexionar sobre un panorama político, legislativo y mediático que ha conseguido generar un marco de conocimiento general sobre el problema pero que, sin embargo, está empezando a ser cuestionado por insuficiente: faltan medidas, falta seguridad, hay que cuidar y mejorar los mensajes informativos. Son estas algunas de las claves del cambio que han sido expuestas por los propios encuestados.

\section{Referencias bibliográficas}

ANDALUCÍA. «Ley 13/2007, de 26 de noviembre, de Medidas de Prevención y Protección Integral contra la Violencia de Género». Boletín Oficial de la Junta de Andalucía 247 (2007), pp. 7-29.

Ander-EGG, Ezequiel. Técnicas de Investigación Social <23. ${ }^{a}$ edición>. Buenos Aires, Magisterio del Río de La Plata, 1993.

BARRedo, Daniel y Oller, Martín. Género, ideología y prensa digital: la construcción informativa del Día Internacional de la Mujer Trabajadora en www.abc.es y www.elpais.com (2001-2010). Alicante, Revista Mediterránea de Comunicación, 2012.<http://goo.gl/Idqj3u>, consultado el 07-05-2014.

BERNARD, H. Russell. Social Research Methods. Qualitative and Quantitative Approaches $<2$. $^{a}$ edición $>$. Londres, Sage, 2013.

32. LUNA DEL CASTILLO, Juan. Informe preliminar sobre la distribución espacio-temporal de los feminicidios habidos en España entre los años 2003 al 2010, 2011. <http://goo.gl/ bDlRrl>, consultado el 13-04-2012.

SANMARTín, José; IbORRA, Isabel; GARCía, Yolanda, y MARTíneZ, Pilar. III Informe Internacional. Violencia contra la mujer en las relaciones de pareja (estadísticas y legislación). Valencia, Centro Reina Sofía, 2010.

Vives-CASES, Carmen; Torrubiano, Jordi y Álvarez, Carlos. «The effect of television news items on intimate partner violence murders», European Journal of Public Health 19 (2009), pp. 592-596. 
BOSCH FIOL, Esperanza y FERRER PÉREZ, Victoria A.: «Nuevo mapa de mitos sobre la violencia de género en el siglo XXI». Psicothema 24, 4 (2012), pp. 548-554.

Coolican, Hugh. Métodos de investigación y estadística en Psicología. México, El Manual Moderno, 1994.

De Miguel, Ana. «Feminismo y juventud en sociedades formalmente igualitarias». Revista de Estudios de Juventud 83 (2008), pp. 29-45.

ESPAÑA. «Ley Orgánica 1/2004, de 28 de diciembre, de Medidas de Protección Integral contra la Violencia de Género». Boletín Oficial del Estado 313 (2004), pp. 42166-42197.

GUTIÉRREZ - RAVÉ, Carmen María. «Encuesta a las alumnas sobre la violencia de género». Innovación y Experiencias Educativas 16 (2009), pp. 1-8. <http://goo.gl/wGuwyy>, consultado el 07-05-2014.

Hernández Rodríguez, Óscar. Estadística elemental para Ciencias Sociales. San José, Universidad de Costa Rica, 2004.

Libera, Irene; Zurbano, Belén y BARredo, Daniel. «El concepto social de la violencia de género en España. Aproximaciones a los imaginarios de jóvenes estudiantes (2013)», Revista interdisciplinaria Estudios de Género de El Colegio de México 2 (2015) (en impresión).

Lozano, José Carlos. Teoría e investigación de la comunicación de masas <2. edición>. México, Pearson Educación, 2007.

LUNA DEL CASTILLO, Juan. Informe preliminar sobre la distribución espacio-temporal de los feminicidios habidos en España entre los años 2003 al 2010, 2011. <http:// goo.gl/FMrOKf>, consultado el 13-04-2012.

Ministerio de IGUALDAd y Universidad Complutense. Igualdad y Prevención de la Violencia de Género en la Adolescencia. Madrid, Ministerio de Sanidad, Política Social e Igualdad, Centro de Publicaciones, 2010. <http://goo.gl/ nU8nZJ>, consultado el 27-11-2013.

Ministerio de Sanidad, Servicios Sociales e Igualdad. Encuesta de opinión sobre la violencia de género. Madrid, Ministerio de Sanidad, Servicios Sociales e Igualdad, Centro de Publicaciones, 2011.<http://goo.gl/ijg278>, consultado el 03-12-2013.

Ministerio de Sanidad, Servicios Sociales e Igualdad. Análisis de la encuesta sobre percepción social de la violencia de género. Madrid, Ministerio de Sanidad, Servicios Sociales e Igualdad, Centro de Publicaciones, 2013. <http://goo.gl/ ndDxbt>, consultado el 04-06-2014.

NACIONES UNIDAS. Declaración sobre la eliminación de la violencia contra la mujer [A/RES/48/104], 1993.

SAnMartín, José; Iborra, Isabel; García, Yolanda y Martínez, Pilar. III Informe Internacional. Violencia contra la mujer en las relaciones de pareja (estadísticas y legislación). Valencia, Centro Reina Sofía, 2010. 
Vives-CaSes, Carmen; TORRubiano, Jordi y Álvarez, Carlos. «The effect of television news items on intimate partner violence murders». European Journal of Public Health 19 (2009), pp. 592-596.

ZURBANO, Belén. «El concepto violencia de género en la prensa diaria nacional española». Cuestiones de Género: de la igualdad y la diferencia 7 (2012), pp. 25-44.

Zurbano, Belén. Guía para la comunicación de la violencia de género. Fuerzas y cuerpos de seguridad. Consejería de Justicia e Interior, Sevilla, 2014.

ZuRBANO, Belén y LiBERIA, Irene. «Revisión teórico-conceptual de la violencia de género y de su representación en el discurso mediático. Una propuesta de resignificación». ZER: Revista de Estudios de Comunicación 36 (2014), pp. 121-143.

ZuRBANO, Belén; LiBERIA, Irene y CAMPOS, Beatriz. «Concepto y representación de la violencia de género. Reflexiones sobre el impacto en la población joven». Oñati Socio-Legal Series. OSLS 5, 2 (2015) (En impresión). ISSN: 2079-5971. 